\title{
LONG-STANDING INTRABRONCHIAL FOREIGN BODIES
}

\author{
BY \\ J. S. A. LINTON \\ From the London Chest Hospital, London, E.2
}

(RECEIVED FOR PUBLICATION FEBRUARY 22, 1957)

The long-standing intrabronchial foreign body presents problems of diagnosis and treatment which are very different from those associated with the recently inhaled foreign body. There may be no history to suggest the original incident ; the clinical picture is usually clouded by superadded pathological changes-atelectasis, pneumonitis, bronchiectasis, or lung abscess-and the bronchoscopic appearances are often misleading. Even when correctly diagnosed, the choice of treatment is not always easy.

Sixteen such cases are presented in an attempt to find common characteristics, recognition of which may assist correct diagnosis in this not uncommon condition.

In addition, the problems of treatment are considered.

\section{CASE Histories}

CASE 1.-A man aged 31 was admitted with 10 months' history of cough with purulent sputum and no haemoptysis. He was febrile $\left(102^{\circ} \mathrm{F}\right.$.), producing $4 \mathrm{oz}$. of purulent sputum daily. Physical and radiological signs were those of consolidation of the right lower lobe. With antibiotics and postural drainage he became afebrile in four days, and there was some radiological resolution. Bronchoscopy showed that the mucosa of the right lower lobe was hyperaemic, granular, and exuding pus. A bronchogram showed the basal segmental bronchi crowded, but not bronchiectatic.

He was discharged after three weeks and followed up as an out-patient. Physical and radiological signs of infection persisted and he lost weight. He was readmitted two years after discharge. He was afebrile (having had antibiotics as an out-patient) with mucoid sputum. A radiograph showed diffuse opacity of the right lower lobe. Bronchography showed contraction of the right basal segments with incomplete filling (Fig. 1).

At bronchoscopy the appearances were unchanged, but in addition a white object was seen which could not be removed. Biopsy of the mucosa showed intense chronic inflammation of the subepithelial layers and haemorrhagic granulation tissue.

At operation the right lower lobe bronchus was incised and a rabbit vertebra removed. The basal segments looked and felt normal. The vertebra lay i so that its canal acted as an airway.

His post-operative course was uneventful. The $\stackrel{\curvearrowright}{\oplus}$ radiograph cleared and a subsequent bronchogram showed no bronchiectasis (Fig. 2).

No history of inhaling the foreign body was obtainable.

CASE 2.-A woman aged 57 was admitted with four years' history of cough, purulent sputum, and wheeziness. There was occasional slight haemoptysis. A febrile incident had confined her to bed for six weeks. On admission she was afebrile and producing $4 \mathrm{oz}$. of mucopurulent sputum daily, containing no malignant cells. There were moist sounds throughout both lung fields. A radiograph showed collapse of the left lower lobe with a calcified shadow at the left hilum, which later disappeared. Bronchography (Fig. 3) showed unfilled basal segments with no bronchiectasis. Bronchoscopy showed pus welling up from the lower lobe. An irregular "tumour" was seen obstructing the basal segments. Biopsy showed squamous metaplasia. Bronchoscopy was repeated. The "tumour" was seen to be granulation tissue, beyond which a bone was seen and was removed.

A subsequent bronchogram (Fig. 4) showed no abnormality.

No history of inhaling the bone was obtainable.

CASE 3.-A man aged 66 was admitted with three years' history of cough and purulent sputum, which was often blood-stained. He was febrile with signs of collapse and consolidation of the right middle and lower lobes. The pyrexia subsided with antibiotics and physiotherapy, but there was no radiological improvement. Bronchoscopy showed granulations in the right lower lobe, but the biopsy was negative. The middle lobe reaerated but not the lower lobe. Bronchoscopy was repeated and a portion of wishbone was removed from the right lower lobe. The right lower lobe subsequently reaerated.

On direct questioning the patient's wife recalled that he had had a choking attack during a meal several years before.

CASE 4.-A girl aged 9 gave a history of many months of cough, which had recently become more troublesome with mucopurulent sputum, loss of weight, and anorexia. She remembered swallowing a collar stud when she was much younger. Her mother stated there had been no metal studs in the house for 


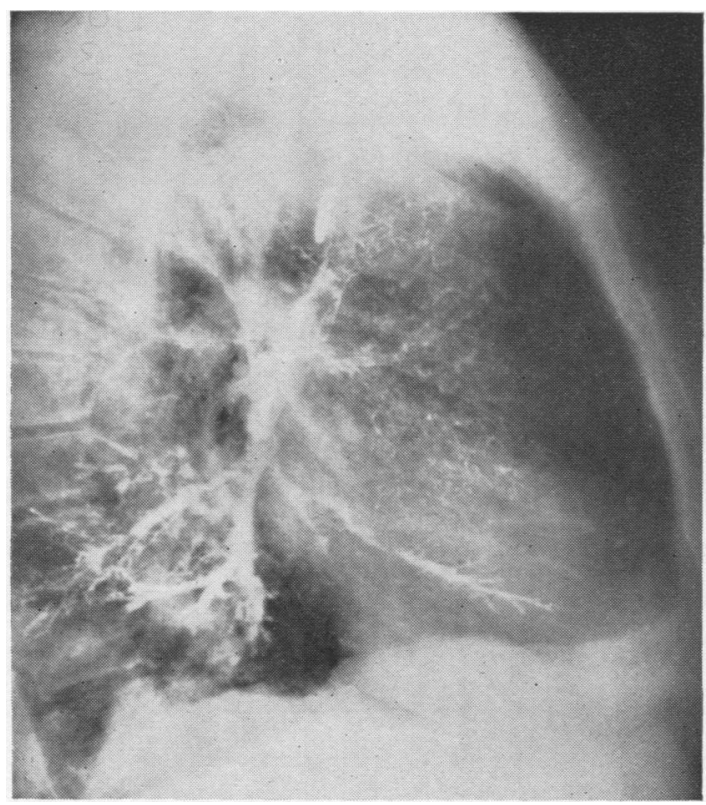

FIG. 1.-Case 1: Pre-operative bronchogram showing contraction of the right lower lobe with incomplete filling of lower obe bronchi.

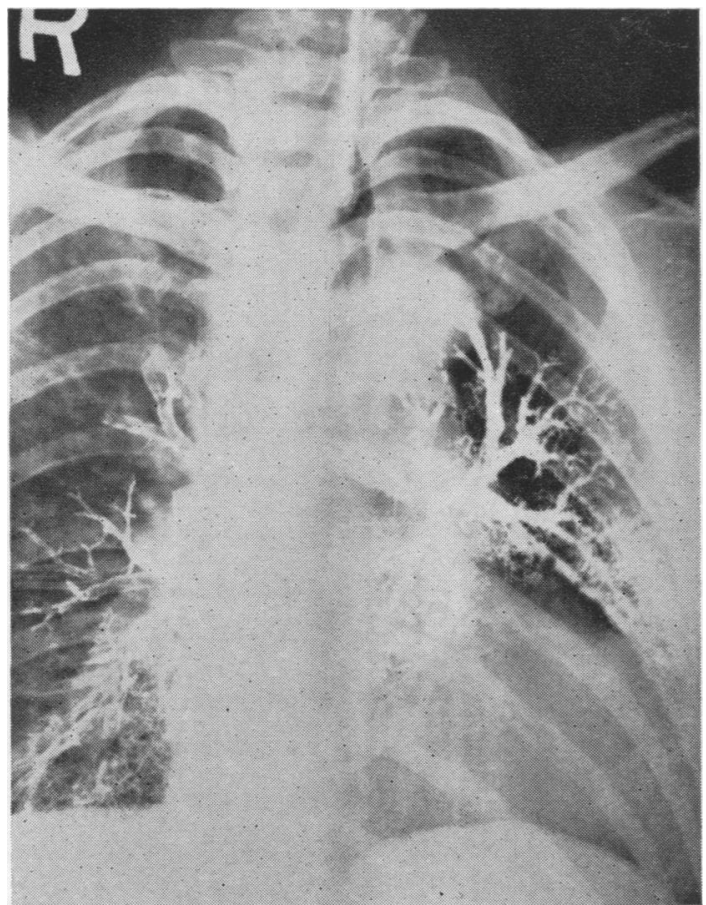

Fig. 3.-Case 2: Pro-bronchoscopy bronchogram showing that the left basal sogments have not filled:

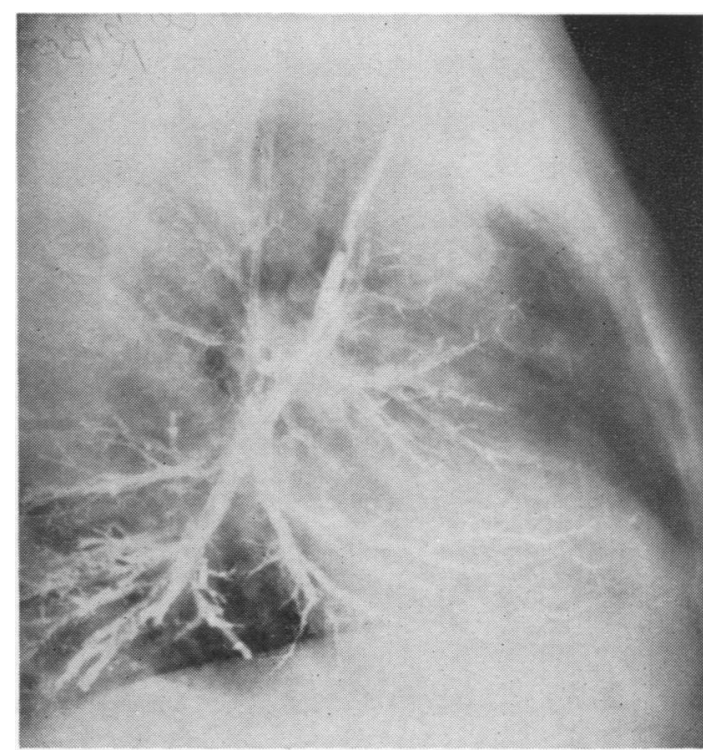

Fig. 2.-Case 1: Post-operative bronchogram showing good filling of the right lower lobe with no bronchiectasis.

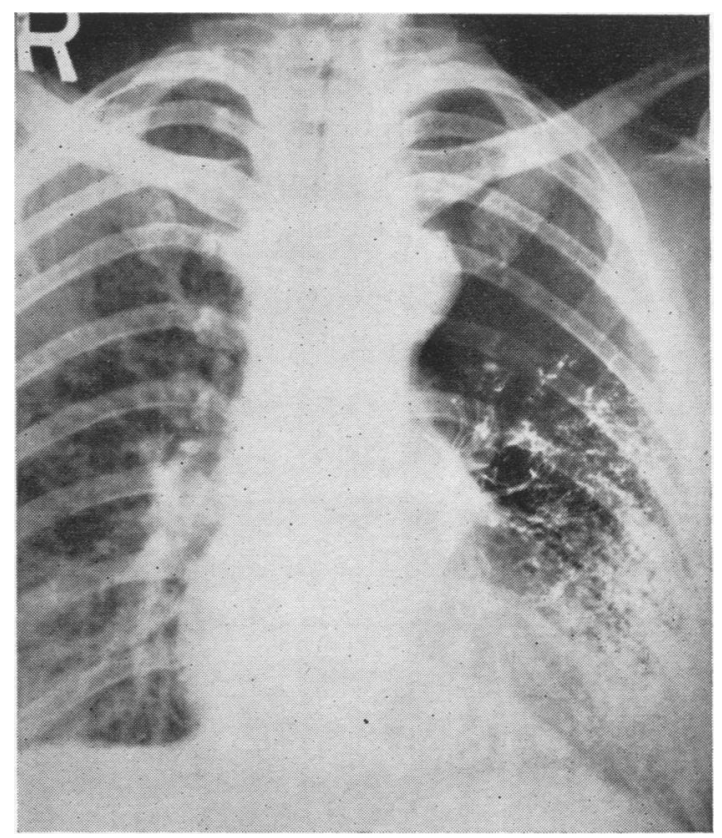

FiG. 4.-Case 2: Post-bronchoscopy bronchogram showing the left lower lobe filled, with no bronchiectasis. 


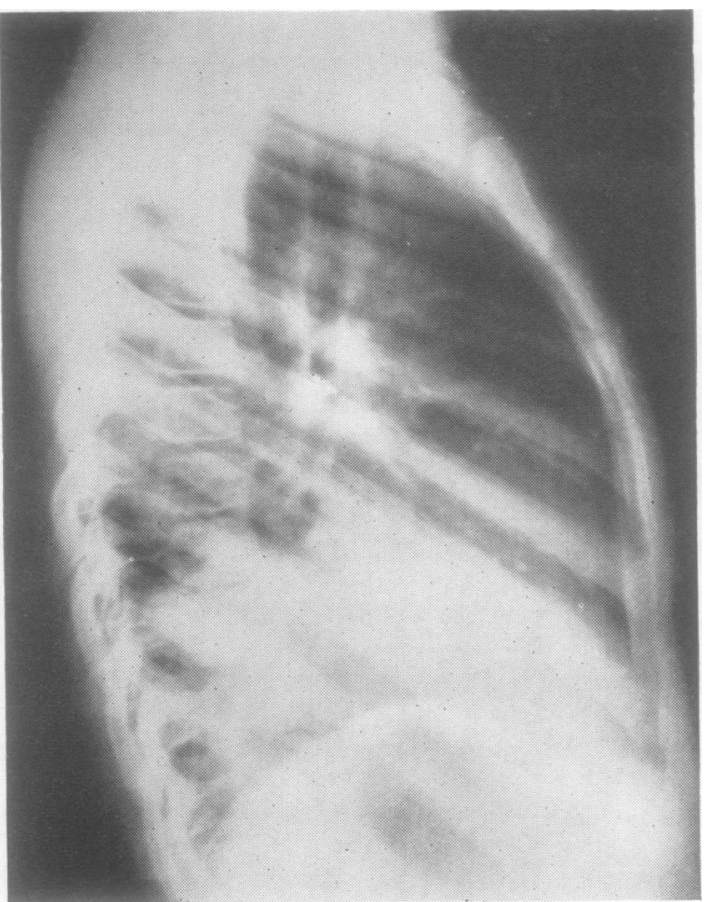

FIG. 5.-Case 4: Pre-bronchoscopy radiograph showing collar stud in the right main bronchus with consolidation of the right basal segments.

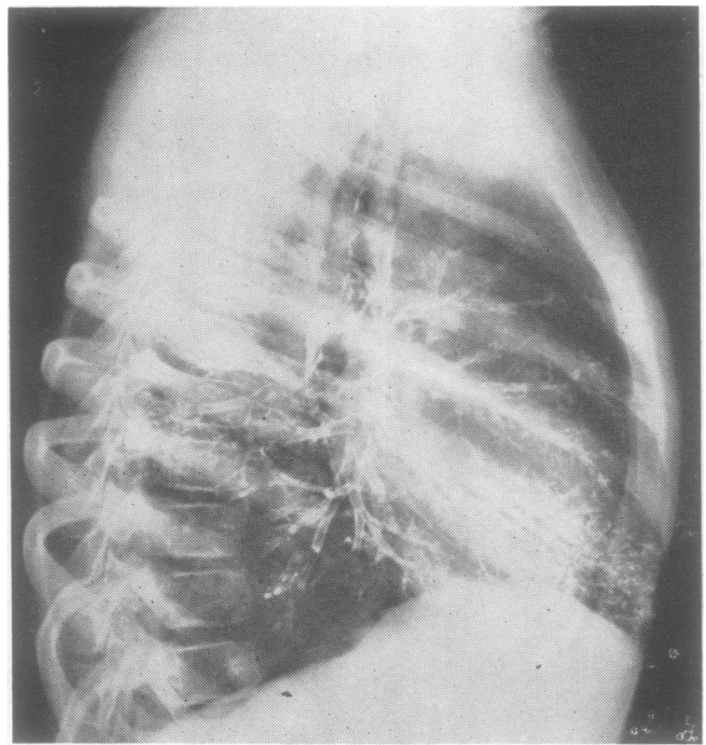

Fig. 6.-Case 4: Bronchogram two weeks after bronchoscopic removal of a foreign body, showing no bronchiectasis.

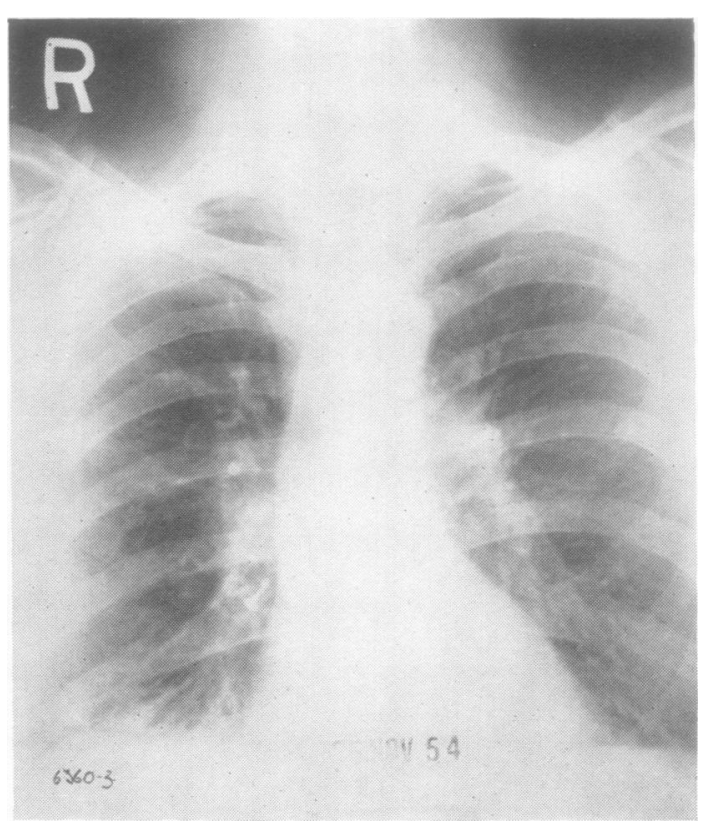

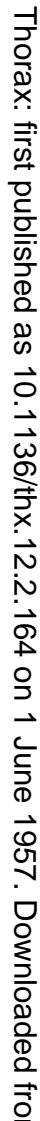

Fig. 7.-Case 7: Radiograph showing a tin-tack in the right lower $\frac{0}{3}$ lobe

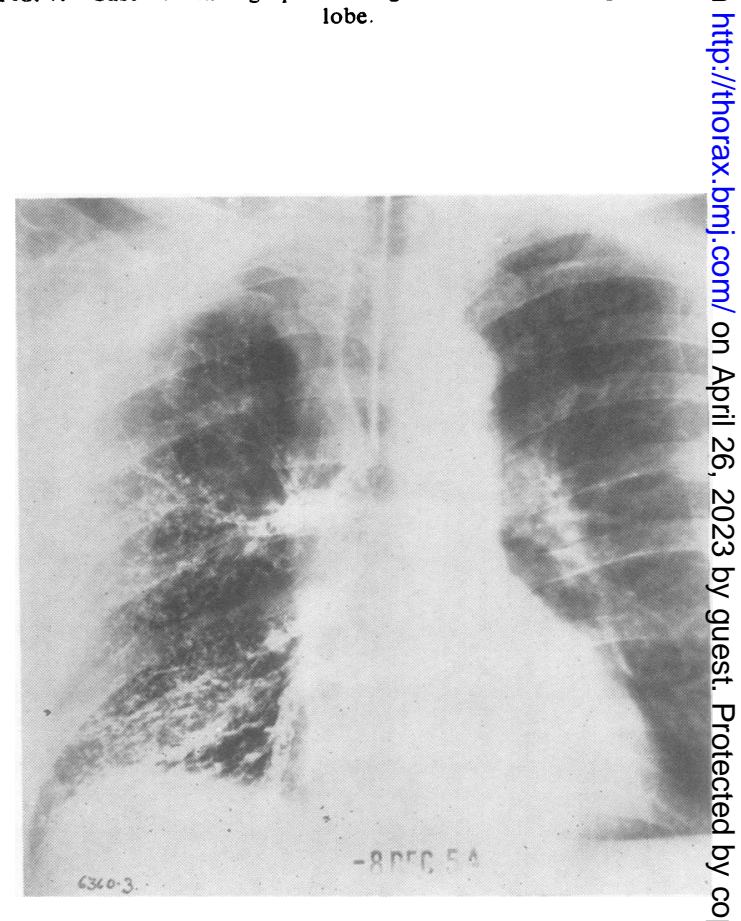

Fig. 8.-Case 7: After expectoration of the tin-tack a bronchogram shows minor bronchiectasis in the posterior basal segment. 
at least five years. A radiograph (Fig. 5) showed the collar stud at the right hilum, with consolidation of the basal segments. At bronchoscopy this was removed from the right lower lobe bronchus. There was some stenosis and considerable granulation tissue. A bronchogram two weeks later (Fig. 6) showed no abnormality.

CASE 5.-A woman aged 48 had been in another hospital six months before with cough, stridor, and purulent sputum, occasionally blood-stained. A thyroid adenoma was found and removed, but cough and sputum persisted. A radiograph showed consolidation of the right upper lobe. At bronchoscopy a large piece of bone was found in the right main bronchus opposite the upper lobe with surrounding granulation tissue. It was easily removed, and there was rapid resolution of the radiological opacity with disappearance of symptoms.

She subsequently gave a history of choking on a mutton bone 18 months before.

CASE 6.-A boy aged 8 gave a history of seven months' irritating cough, and developed left-sided pneumonia. A radiograph showed a metallic foreign body, whose nature was obscure, with atelectasis of the left lower lobe. Bronchoscopy revealed a shining metallic object in the left main bronchus, opposite the upper lobe orifice. Beyond its presenting rounded surface was a mass of granulation tissue. On removal it proved to be a note-book pencil with flanged metal end.

The child became symptom-free, but was lost sight of, and no follow-up is recorded.

CASE 7.-A man aged 55 gave a history of inhaling a tin-tack three months previously, with subsequent cough and mucopurulent sputum, never blood-stained. A radiograph (Fig. 7) showed the tin-tack in the right lower lobe. Before investigations could be started the patient expectorated the tack and has been symptom-free ever since. Subsequent bronchography (Fig. 8), however, showed minor bronchiectasis of the posterior basal segment.

CASE 8.-A girl aged 11 had bilateral bronchiectasis with symptoms since infancy. When aged 5 , middle lobectomy was performed. After post-operative bronchoscopy, it was suspected that part of the rubber sucker tip might have been left in the bronchial tree, but a search was unsuccessful. Cough and sputum persisted, and she was thought to have chronic bronchitis, which was improved by a prolonged course of terramycin. A bronchogram, six years after operation, showed bronchitic changes in the right lower lobe, with minimal bronchiectasis. Two weeks later she coughed up a piece of rubber tube $8 \mathrm{~mm}$. long, and since then, with no treatment, has had no further symptoms.

CASE 9.-A woman aged 54 gave a history of inhaling a peanut seven months previously, and had had cough and purulent sputum, with occasional haemoptysis, ever since. A radiograph showed col- lapse and consolidation of the right middle and lower lobes, and a bronchogram demonstrated bronchiectasis of these lobes. Bronchoscopy showed reddening and purulent secretion in the middle and lower lobes, but no foreign body. She was treated by resection of the diseased lobes. At pre-operative bronchoscopy a whole peanut was removed by the sucker. The specimen showed bronchiectasis and pneumonitis with ulceration at the origin of the apical lower bronchus.

CASE 10.-A man aged 40 inhaled a tin-tack two and a half years before admission. He had had cough and purulent sputum ever since with repeated haemoptysis. A radiograph showed the tack in the right lower lobe. A bronchogram showed poorly filled, bronchiectatic basal segments with the foreign body in the posterior basal segment. At bronchoscopy the tack could not be seen. He was treated by right lower lobectomy. The specimen showed severe bronchiectasis, wih fibrosing collapse and fibrous thickening of the septa and pleura.

CASE 11.-A boy aged 8 gave a history of cough and purulent sputum since a rather indefinite incident of inhaling food one year before. A radiograph showed consolidation of the right basal segments. Bronchography showed poor filling of these segments but no definite bronchiectasis. At bronchoscopy the right lower lobe was seen to be obstructed by a lump of purulent material, which was removed by suction, causing some bleeding. Treatment was right lower lobectomy. The specimen showed a foreign body in a basal bronchus which had caused bronchiectasis, infection, fibrosis, and collapse.

CASE 12.-A woman aged 43 had aspirated a tooth into the right lung during a dental extraction under general anaesthesia six years previously. She had been bronchoscoped several times, but the tooth could not be removed. She developed cough, purulent sputum, and haemoptysis. A radiograph showed collapse of the right basal segments with a calcified shadow, presumed to be the tooth. Bronchography showed poorly filled, contracted basal segments with slight bronchiectasis. At operation the tooth was felt in the posterior basal segment. It was removed by bronchotomy, but the basal segments remained solid and airless and were therefore removed. The specimen showed bronchiectasis with peribronchial fibrosis.

CASE 13.-A man aged 66 had had cough and sputum for years, worse for nine months, with recent haemoptysis. He was admitted to another hospital and treated with penicillin for collapse and infection of the right lower lobe. Bronchoscopy showed nodular friable " growth" in the right lower lobe, but biopsy was reported as squamous metaplasia. He was admitted as a case of bronchial carcinoma. No malignant cells were found in the sputum and radiographs showed reaeration of the previously collapsed lobe, though with residual patchy opacities. Bronchoscopy was repeated and showed the right lower lobe bronchus reddened and narrowed, with friable nodular projections. Biopsy was negative. He developed pyrexia 
which subsided with penicillin. A bronchogram showed absent filling of the right basal segments with over-expansion of the middle and upper lobes. Treatment was right lower lobectomy for a presumed carcinoma. The specimen showed a piece of bone embedded in the basal bronchi with ulceration of the epithelium and extreme squamous metaplasia.

No relevant history was obtainable.

CASE 14.-A man aged 54 had had right-sided chest pain, loss of weight, and mucopurulent sputum for five months. A radiograph showed consolidation and cavitation of the right upper lobe. The sputum contained no malignant cells. Bronchoscopy was negative. A bronchogram was technically poor, with little filling of the right upper lobe. Carcinoma was diagnosed. At operation there was a mass in the right upper lobe, possibly extending into the middle lobe, with enlarged hilar lymph nodes. The upper and middle lobes were resected. The specimen showed the apical segment of the upper lobe to be fibrotic, with areas of necrosis and partial excavation. Sections of the abscess showed purulent material surrounded by fibrosis. Other material was present which appeared to be the remains of a foreign body. Other portions showed areas of pneumonia and collapse.

No relevant history could be obtained.

CASE 15.-A woman aged 52 had had cough with blood-stained purulent sputum for six months. A radiograph showed an opacity at the apex of the left lower lobe, with a small calcified object, appearing like a calcified lymph node. One specimen of sputum out of seven had cells suspicious of carcinoma. Bronchoscopy was negative. At operation an irregular, hard mass was present in the apex of the left lower lobe. Lobectomy was performed. The specimen showed a firm, pale area, $1 \frac{1}{2}$ in. in diameter, lying peripherally, with the crown of a carious tooth occluding the bronchus. Section showed a chronic lung abscess. The patient later stated that she had had a dental extraction under general anaesthesia nine years previously.

CASE 16.-A man aged 52 had right-sided pneumonia 18 months previously, with persistent cough and foul sputum ever since and occasional haemoptysis. Bronchoscopy at another hospital showed no abnormality except pus in the right main bronchus. A radiograph showed a large abscess in the apex of the right lower lobe. The sputum contained no malignant cells. Bronchoscopy was repeated and showed pus in the right lower lobe with a large slough, which was removed. His general condition improved, but the abscess remained. At operation the pleura was densely adherent and the fissures fused. For technical reasons pneumonectomy was performed. The specimen showed the lobe largely replaced by an abscess, surrounded by collapsed fibrotic lung. The contents were purulent and contained a vegetable pea. The appearances suggested that this was aspirated into the apical bronchus of the lower lobe.

No relevant history was obtainable.

\section{Discussion}

History.-Of these 16 cases, only five volun $\frac{\bar{क}}{\bar{\Phi}}$ teered a history of inhaling a foreign body. Five recalled the incident only after the foreign bodyen had been removed. In the other six no relevant history was obtainable, even in retrospect.

Where it was possible to assess the length o $\overrightarrow{\mathrm{w}}$ time between inhalation and removal of the foreign body, this varied from nine years to six months.

The presenting symptoms were those of the resulting pathological changes. Cough and puru $\vec{\Phi}$ lent sputum were constant. Febrile incidents wereo common. In several cases the symptoms did not appear for some time after the original obstruction occurred and were readily relieved by5 symptomatic treatment with antibiotics and physiotherapy, only to recur at intervals until the foreign body was eventually removed. Jackson $>$ and Jackson (1950) comment on these symptomless intervals as a source of error in diagnosis.

Haemoptysis occurred in nine cases; it waso severe in three. Moore (1951) makes the pointo that "simple" bronchiectasis seldom produces $\frac{D}{\varrho}$ haemoptysis, but that it is common with bronchi- $\vec{\Rightarrow}$ ectasis caused by foreign bodies. Laurance (1954) considers haemoptysis unusual with foreign? bodies.

INVESTIGATION.-As might be expected, the rightō side (13 cases) was affected more often than the left (three cases) and the lower lobe more com- $-\overline{0}$ monly than the upper.

Physical examination indicated the super-ô imposed pathological lesion. There is no record of the detection of a localized wheeze.

A plain radiograph revealed the foreign bodies $D$ where these were metallic. Bone fragments and tooth fragments were sometimes visible in retro- No spect when the opacities were noticed to have disappeared. At first sight they appeared simply as 0 parahilar calcification, indistinguishable from $\omega$ calcified tuberculous lymph nodes. Glennie, Dark,, and Lindars (1952) had the same experience. The appearances otherwise were those of atelectasis, $\underset{\varnothing}{\varnothing}$ pneumonitis or abscess, with no indication of the cause.

Bronchography, when performed, was in no $\frac{\mathrm{O}}{\mathrm{D}}$ case helpful in making the diagnosis. It merely $\frac{?}{\mathbb{Q}}$ confirmed the presence of bronchiectasis, atelec- $\varrho$ tasis, or bronchial obstruction.

Bronchoscopy should have been conclusive 8 where the diagnosis was still in doubt. The characteristic finding, even when the foreign body 
itself cannot be seen, is the presence of heaped-up, friable granulation tissue, surrounding the foreign body and often obscuring it. This appearance is probably well known and is recorded in most papers on the subjest (Jackson and Jackson, 1950 ; Laurance, 1954 ; Moore, 1951). It is unfortunate that, in five cases, the significance of this finding was not appreciated by experienced bronchoscopists who, in each case, made the diagnosis of carcinoma. Where biopsy material was obtained this showed normal mucosa, granulation tissue, or squamous metaplasia. This coincidence of a carcinoma-like bronchoscopic appearance with a biopsy showing inflammatory changes should certainly have suggested a diagnosis of foreign body. In three of these five cases bronchoscopy was repeated and established the diagnosis.

The superadded pathological changes were those of atelectasis and pneumonitis in 10 cases, bronchiectasis (demonstrated by bronchography) in three, and chronic lung abscess in three. Jackson and Jackson (1950) maintain that bronchiectasis is a rare sequel of exogenous foreign body, contrary to statements in the literature, and that these statements are made chiefly on the basis of foul sputum without corroboration by bronchography. They are also of the opinion that true lung abscess is rare, though "drowned lung" can occur which recovers after removal of the foreign body. Laurance (1954) also considers that lung abscess is an uncommon sequel to inhaled foreign body. Thomson and Negus (1955) probably speak nearest to the truth when they say that bronchial abscess is to be expected after impaction of a foreign body. They also stress that a bronchial abscess will heal without permanent damage to the lung after the bronchial obstruction has been relieved.

Twelve patients were of an age when carcinoma should be suspected, and in six cases this was the provisional diagnosis on admission. Three came to operation with this diagnosis. Sputum was examined for malignant cells in eight cases-an investigation found to be of considerable significance in this hospital (Jennings and Shaw, 1953): all were negative except in one case, where one specimen in seven was reported as showing cells suspicious of carcinoma, and this was from another hospital.

At the end of these investigations foreign body had been correctly diagnosed in 11 of the 16 cases. Carcinoma remained the presumed diagnosis in three patients. One was thought to have residual bronchiectasis with bronchitis and one a simple chronic lung abscess.
Treatment.-As previously mentioned, the choice of treatment is not always easy even after correct diagnosis.

Of the incorrectly diagnosed cases in this series, three were thought to be malignant and had resections, the foreign body being identified only on examination of the specimen. The case diagnosed as lung abscess was also treated by resection. The patient thought to have residual bronchiectasis coughed up her foreign body after six years and has been symptom-free ever since.

Of the foreign bodies correctly diagnosed (11), one was coughed up; five were extracted at bronchoscopy; one was removed by bronchotomy ; and four were resected.

All the patients treated without resection had complete relief of symptoms and resolution of physical and radiological signs, although their average length of history was three years. Where bronchography was performed at a later date (four cases), only one showed bronchiectasis and this was of minor degree.

Jackson and Jackson (1950) state that the prognosis is good after removal of the foreign body, even after long sojourn. In more than 200 of such cases in their report, all recovered complete health except four. They noted that foreign bodies of vegetable origin caused more rapid permanent damage than relatively inert material such as metal, teeth, and bone. Weinberg $(1937,1938)$ agrees with this and explains it on the grounds that these foreign bodies, by virtue of their rigidity and irregular shape, are less likely to cause early complete obstruction of the bronchus. In his experiments with vegetable foreign bodies, artificially introduced into the bronchi of rabbits, he found obstructive emphysema occurring in the first 20 minutes, infection and collapse in six to 42 hours, followed by frank bronchiectasis in 14 to 58 days. Inert foreign bodies (teeth, tacks) produced first ulceration at the site of impaction, then infection leading to complete obstruction with atelectasis and bronchiectasis. The extent of the damage varied with the degree of obstruction and the pathogenicity of the organisms present. Incomplete obstruction produced patchy atelectasis and bronchial dilatation of variable degree.

These statements are partially supported by the findings in our 16 cases. The correctly diagnosed foreign bodies treated by resection (four) had irreversible bronchiectatic changes. Of the foreign bodies treated without resection, one was a tin-tack which had produced incomplete obstruction, though with minor bronchiectasis ; four were bone fragments; one was a rubber sucker tip, one 
a metal-tipped pencil, and one a metal collar stud. These, although causing ulceration with infection and granulation tissue, had not produced irreversible damage, in spite of long histories. The obstruction was demonstrably incomplete in three cases, but appeared complete in the others.

Cooley, Ginsberg, Olsen, and Kirklin (1956) state that surgical resection is the treatment of choice when the condition has already developed and gives excellent results.

Permanent, irreversible lung damage may not occur even with obvious infection and apparently complete obstruction. It is therefore suggested that simple removal of the foreign body by bronchoscopy or bronchotomy should suffice in many cases which are at present treated by resection. Neither the length of history, the degree of radiological abnormality, nor the presence of profuse, purulent sputum is an absolute contraindication to an attempt at preserving lung tissue. Only when obvious irreversible changes have occurred, such as true lung abscess or gross bronchiectasis with fibrosis and parenchymal destruction, is it necessary to resect the affected part.

\section{SUMMARY}

Sixteen cases of "chronic" intrabronchial foreign body are reported. The difficulties of diagnosis are outlined and an attempt is made to find common features in these cases. Consideration of the possibility of the condition is probably the most important factor in arriving at the correct diagnosis. The possible lines of treatment are discussed, with particular reference to the desirability of conserving lung tissue when this has not suffered permanent damage.

My thanks are due to Mr. T. Holmes Sellors, Mr. V. C. Thompson, Mr. D. Barlow, and Mr. J. R. Belcher for permission to publish their cases, to Dr. $\mathrm{K}$. Hinson for the pathological reports, and to $\mathrm{Mr}$. D. Kemp, of the Brompton Hospital, for preparation of the illustrations.

\section{REFERENCES}

Cooley, J. C., Ginsberg, R. L., Olsen, A. M., and Kirklin, J. W. (1956). J. thorac. Surg., 31, 615.

Glennie, J.S., Dark, J. F., and Lindars, D. C. (1952). Brit. med. J., 1,1009 .

Jackson, C., and Jackson, C. L. (1950). Bronchoesophagology, pp. 16, 20,106, 107, and 175. Saunders, Philadelphia.

Jennings, R. C., and Shaw, K. M. (1953). Thorax, 8, 288.

Laurance, B. (1954). Brit. med. J., 1, 125.

Laurance, B. (1954). $\quad \begin{aligned} & \text { Brit. med. J., } \\ & \text { Moore, B. P. (1951). }\end{aligned}$

Thomson, St. Clair, and Negus, V. E. (1955). Diseases of the Nose and Throat, 6th ed. Cassell, London.

Weinberg, J. (1937). J. thorac. Surg., 6, 402.

- (1938). Tbid., 7, 488. 